Original Research Paper

\title{
Enhanced Decision Support System Applications Based on Complex Intuitionistic Fuzzy Soft Expert Sets Theory
}

\author{
${ }^{1}$ Malek Alksasbeh, ${ }^{2}$ Mohammad Al-kaseasbeh, \\ ${ }^{3}$ Saba Al-Kaseasbeh, ${ }^{3}$ Khalid Alemerien and ${ }^{1}$ Bassam A. Alqaraleh \\ ${ }^{I}$ Faculty of Information Technology, Al-Hussein Bin Talal University, Ma'an, Jordan \\ ${ }^{2}$ Faculty of Science, Jerash University, Jerash, Jordan \\ ${ }^{3}$ College of Science, Tafila Technical University, Tafila, Jordan
}

Article history

Received: 15-08-2017

Revised: 26-09-2017

Accepted: 27-11-2017

Corresponding Author:

Malek Alksasbeh,

Faculty of Information

Technology, Al-Hussein Bin

Talal University, Ma'an, Jordan

Email: malksasbeh@gmail.com

\begin{abstract}
Decision making is one of the important milestones in the development of various real-life aspects. Decision Support System (DSS) is a computerized information system used to enhance decision-making in several areas. This study aims to improve DSS applications by extending two concepts of complex intuitionistic fuzzy sets and soft expert sets to propose the theory of complex intuitionistic fuzzy soft expert sets and defines some related concepts pertaining to this notion as well as the basic operations on this concept. Moreover, the algorithm for such concept is developed.
\end{abstract}

Keywords: Decision Support System, Complex Fuzzy, Soft Expert Set

\section{Introduction}

The development of computer information systems and the related issues of automating human reasoning and inference have raised a challenge to researchers. Uncertainty is among the most real-life problems which seriously require new concepts, models and systems (Karacapilidis, 2006). DSS is a computer-based information system which developed to provide a systematic decision regarding uncertainty problems (Power and Sharda, 2009).

Several classical mathematical models and theories have been used as tools for DSS such as fuzzy set theory (Zadeh, 1965). Two decades later, Atanassov (1986) introduced Intuitionistic Fuzzy Set (IFS) as an extension of Zadeh's notion of the fuzzy set to deal with vagueness and uncertainty as a controlling tool. They are wellknown mathematical tools for supporting the solution of DSSs (Jemal et al., 2017; Zimmermann, 1998). However, they are facing many challenges dealing with uncertainties and fuzziness (Molodtsov, 1999). In order to overcome those challenges, Molodtsov (1999) introduced the theory of soft sets. He also demonstrated that the soft set theory can be applied in solving many practical problems in physics, economics and engineering, among others. Later, Maji et al. (2001) extended the study of soft sets by initiating the concept of fuzzy soft sets and applied these theories in solving various Decision Making (DM) problems. Further, Young et al. (2004) improved the theory of soft sets by introduced Intuitionistic Fuzzy Soft Set (IFSS) theory which combines the advantages of soft set and IFS.

A large number of real-life problems are complicated and cannot be modeled using one-dimensional classes and/or one-dimensional variables. To deal with such problems, Ramot et al. (2002) introduced Complex Fuzzy Sets (CFS) which characterized by membership function assign with the complex-valued grade. CFS has been used to solve various problems such as the multiple periodic factor prediction problems (Jun et al., 2012). Currently, research on CFS is moving rapidly. Complex Intuitionistic Fuzzy Set (CIFS) generalized CFS by adjoining complex-valued nonmembership with the complex-valued membership for all it is objects (Alkouri and Salleh, 2013; 2012; Ali et al., 2016).

It is worth to mention that fuzzy set, IFS and CIFS are independent of the parameterization tools whereas soft set, fuzzy soft set and IFSS are parametric in their own nature. Since then, many researchers have studied these theories and created some algorithms to solve problems in DM. Yet, none of the previous algorithms provide an opinion of experts. This causes a problem especially with those who need to consult experts. Soft expert set was introduced by Alkhazaleh and Salleh (2011) as a generalization of soft sets where experts' opinions are taken place.

The main aim of the present paper is to combine CIFS with soft expert set to introduce Complex Intuitionistic Fuzzy Soft Expert Set (CIFSES). Our motivation of such 
combined-rich-concept is to solve complicated DM problems by developing a new algorithm.

\section{Literature Reviews}

This section briefly reviews some research focused on applying fuzzy set and soft expert set to solve the problems in DM.

Alkhazaleh et al. (2011) introduced possibility fuzzy soft set and gave an application of this theory to solve DM problem in terms of medical diagnosis problem. Extending the concept of possibility fuzzy soft set to intuitionistic fuzzy set and soft expert set have been done by Bashir et al. (2012) and Bashir and Salleh (2012b) respectively. They applied their proposed concepts to solve DM problems. Later, Selvachandran and Salleh (2015) combined both concepts of intuitionistic fuzzy set and soft expert to introduce possibility intuitionistic fuzzy soft experts set and applying it in solving DM problems.

Other extensions have been made by involving the concept of interval-valued fuzzy set and interval-valued vague set. These concepts are generalized intervalvalued fuzzy soft set and generalized interval-valued vague soft set were introduced by Alkhazaleh and Salleh (2012) and Alhazaymeh and Hassan (2013) respectively. Neutrosophic-type sets have also attracted the interest to extend many other concepts and applying them in solving DM problems. Few to mentions, possibility neutrosophic soft sets with applications in decision making and similarity measure (Karaaslan, 2014), single-valued neutrosophic soft expert sets and their applications in decision making (Broumi and Smarandache, 2015) and neutrosophic soft expert sets (Sahin et al., 2015).

However, these studies have shown inaccurate steps in their algorithms especially when the universal set contains more than three elements. In fact, they examined universal set with two or three elements only. Eventually, it would lead to inaccurate results in general.

The reason behind that is related with a common inaccurate step in their algorithms. More precisely, the reason is: choosing the highest grade among all the $u$ 's that attached with the same parameter. This might lead to drop some of the $u$ 's who had not obtained the highest grade, but with the best overall performance. As a result, choosing such algorithms shows bias.

\section{Mathematical Preliminaries}

This section begins with some notations of classical set theory. And then, provides some definitions and results, that paving the way for the following two sections.

The symbol $\phi$ denotes the empty set, $A^{c}$ denotes the complement of $A$ with respect to some universal set $U$ $A \cap B$ denotes the intersection of sets $A$ and $B, A \cup B$ denotes the union of sets $A$ and $B$ and $A-B$ denotes the difference of sets $A$ and $B$ whose elements belong to $A$ but not to $B$.

In classical set theory, when element belongs to the set, is fully belongs to the set. Dealing with uncertainty requires a certain degree of belongingness which is not provided in the firm classical set theory. Attaching each element with membership grade, form a more desirable concept. This concept coined by fuzzy set (Zadah, 1965).

In the forthcoming definition, we formally define the fuzzy set over a universal set $U=\left\{u_{i}: i=1, \ldots, p\right\}$.

Definition 1: A fuzzy set $A$ over a universal set $U$ is characterized by a membership function:

$$
\mu_{A}(u): A \rightarrow[0,1]
$$

Symbolically, A fuzzy set $A$ over $U$ can be written as:

$$
A=\left\{\left(u_{i}, \mu_{A}\left(u_{i}\right)\right): u_{i} \in U\right\} .
$$

In some occasions, determining the value of the membership is arguing. Nevertheless, we can deal with uncertainty by parameterzing a set of objects $U$ with their attributes, factors, properties, or any other parameter describes the object $u$ in $U$. We call such parameterization, soft sets. Soft sets were introduced by Molodtsov (1999).

In the next definition, we formally defined soft sets over $U$ with a set of parameters $E=\left\{e_{j}: j=1, \ldots, q\right\}$.

Definition 2: A soft set $(F, A)$ over $U$ is a parameterized family of subsets of $P^{*}(\mathrm{U})$ represents as

$$
F: A \rightarrow \mathcal{P}^{*}(U)
$$

where, $A \subseteq E$ and $P^{*}(U)$ is the set of all nonempty subset of $U$.

Definition 2 follows the notation of Zhu and Wen (2013), who revisited soft sets' operation based on inherited properties and operations from classical sets.

In particular, Definition 2 is slightly different from the Molodstov's one. Molodstov considered the codomain of the soft set as the set of all subsets of $U$ whereas Zhu and Wen considered the codomain of the soft set as the set of all nonempty subsets of $U$.

This restriction seems rational, because if there is a parameter $e \in E$ which does not describe any $u \in U$ in anyhow, then such parameter will not store any knowledge from the soft set and then, should be eliminated.

Fuzzy sets and soft sets have been generalized through the years. Fuzzy set has been generalized to intuitionistic fuzzy set which characterized by 
membership and nonmembership (Atanassov, 1986), complex fuzzy set which characterized by complexvalued membership (Ramot et al., 2002) and complex intuitionistic fuzzy set which characterized by complex valued membership and nonmembership (Alkouri and Salleh, 2013; 2012; Ali et al., 2016). Soft set has been generalized to fuzzy soft set (Maji et al., 2001), intuitionistic fuzzy soft set (Xu et al., 2010) and complex intuitionistic fuzzy soft set (Kumar and Bajaj, 2014).

Alkhazaleh and Salleh (2011) introduced the concept of soft expert set where experts' opinions regarding the knowledge stored in the soft set are taken place. Soft expert set has been generalized rapidly, few researches to mention (Hazaymeh et al., 2012; Bashir and Salleh, 2012a; AlQuran and Hassan, 2016;Al-Qudah and Hassan, 2017).

Now, let us formally recall the definitions of intuitionistic fuzzy set, complex fuzzy set, complex intuitionistic fuzzy set, fuzzy soft set, intuitionistic fuzzy soft set, complex intuitionistic fuzzy soft set and soft expert set.

Definition 3: An intuitionistic fuzzy set $A$ over a universal set $U$ is characterized by a membership function $\mu_{A}(u)$ and nonmembership function $v_{A}(u)$ where

with

$$
\begin{aligned}
& \mu_{A}(u): A \rightarrow[0,1], \\
& v_{A}(u): A \rightarrow[0,1]
\end{aligned}
$$

$$
0 \leq \mu_{A}(u)+\mathrm{v}_{A}(u) \leq 1
$$

Definition 4: A complex fuzzy set $A$ over a universal set $U$ is characterized by a membership function $\mu_{A}(u)$ :

$$
\mu_{A}(u): A \rightarrow D
$$

where, $\mathbb{D}$ is the set of all complex numbers $C$ in the open unite disk, $\mathbb{D}=\{z \in C:|z|<1\}$.

The membership function $\mu_{A}(u)$ of complex fuzzy set can be written exponantial form as $r_{A}(u) e^{i w_{A}(u)}$ where $i=\sqrt{-1}$ and both $r_{A}(u)$ and $w_{A}(u)$ are real-valued. More precisely, the function $r_{A}(u)$ maps to $[0,1]$ and called amplitude function while $w_{A}(u)$ is an arbitrary realvalued and is called phase function. Therefore, complex-valued membership $\mu_{A}(u)$ describes the object $u$ by two parameters; like how strong you need the object $u$ and for how long, or how strong you need the object $u$ and when. The nature of complex fuzzy set working effortlessly with periodic problems.

Definition 5: A complex intuitionistic fuzzy set $A$ over a universal set $U$ is characterized by a membership function $\mu_{A}(u)$ and nonmembership function $v_{A}(u)$ where:

$$
\begin{aligned}
& \mu_{A}(u): A \rightarrow \mathbb{D}, \\
& v_{A}(u): A \rightarrow \mathbb{D},
\end{aligned}
$$

and:

$$
\left|\mu_{A}(u)+v_{A}(u)\right| \leq 1
$$

The following definition combines Definition 1 and Definition 2 of fuzzy set and soft set, respectively.

Definition 6: A fuzzy soft set $(F, A)$ over $U$ is a parameterized family of subsets of $F^{*}(U)$ represents as:

$$
F: A \rightarrow F^{*}(U)
$$

where, $A \subseteq E$ and $F^{*}(U)$ is the set of all nonempty fuzzy subset of $U$

Definition 7: An intuitionistic fuzzy soft set $(F, A)$ over $U$ is a parameterized family of subsets of $I^{*}(U)$ represents as:

$$
F: A \rightarrow I^{*}(U)
$$

where, $A \subseteq E$ and $I^{*}(U)$ is the set of all nonempty intuitionistic fuzzy subset of $U$.

Definition 8: A complex intuitionistic fuzzy soft set $(F$, $A$ ) over $U$ is a parameterized family of subsets of $C^{*}(U)$ represents as:

$$
F: A \rightarrow C^{*}(U)
$$

where, $A \subseteq E$ and $C^{*}(U)$ is the set of all nonempty complex intuitionistic fuzzy subset of $U$.

Let $U$ be a universal set, $E$ a set of parameters and $W$ a set of experts (consultants). Let $O$ be a set of opinions, $Z=E \times W \times O$ and $A \subseteq Z$.

Definition 9: A soft expert set $(F, A)$ over $U$ is a parameterized family of subsets of $P^{*}(U)$ represents as:

$$
F: A \rightarrow P^{*}(U)
$$

where, $A \subseteq Z$ and $P^{*}(U)$ is the set of all nonempty subset of $\bar{U}$. form: 


$$
\left(\left(e_{j}, w_{k}, O\right),\left\{u_{i}: u_{i} \in U\right\}\right)
$$

Where:

$$
W=\left\{w_{k}: k=1, \ldots, k\right\} \text { and } O=\{0,1\}
$$

\section{Complex Intuitionistic Fuzzy Soft Expert Sets}

In this section, we introduce a concept of a complex intuitionistic fuzzy soft expert sets (CIFSES).

To avoid ambiguity, we emphasize the notation of the following definition. Let $U$ be a universal set, $E$ a set of parameters and $W$ a set of experts (consultants). Let $O$ be a set of opinions, $Z=E \times W \times O$ and $A \subseteq Z$.

Definition 10 A complex intuitionistic fuzzy soft expert set $(F, A)$ over $U$ is a parameterized family of subsets of $C^{*}(U)$, represents as:

$$
F: \mathrm{A} \rightarrow C^{*}(U)
$$

where, $C^{*}(U)$ is the set of all nonempty complex intuitionistic fuzzy set over $U$.

In particular, complex intuitionistic fuzzy soft expert set contains elements of the form:

$$
\left(\left(e_{j}, w_{k}, O\right),\left\{\left(u_{i}, \mu_{A}\left(u_{i}\right), v_{A}\left(u_{i}\right)\right): u_{i} \in U\right\}\right)
$$

where the tuple $\left(e_{j}, w_{k}, O\right) \in A \subseteq Z$ assigns with complex intuitionistic fuzzy set $\left\{\left(u_{i}, \mu_{A}\left(u_{i}\right), v_{A}\left(u_{i}\right)\right): u_{i} \in U\right\}$. That is, $F\left(e_{j}, w_{k}, O\right)=\left\{\left(u_{i}, \mu_{A}\left(u_{i}\right), v_{A}\left(u_{i}\right)\right): u_{i} \in U\right\}$ is a complex intuitionistic fuzzy set -valued function.

\section{Operation on CIFSESs}

This section follows Zhu and Wen (2013) approach of defining basic operations of soft sets. Let us begin with the notions of empty and universal complex intuitionistic fuzzy soft expert set. Molodtsov called a soft set $(F, A)$ by null soft set if $F(e)=\phi$ for all $e \in A$. In Zhu and Wen approach, $\phi$ does not belong to the codomain of $F$. Thus, we define the concept of empty complex intuitionistic fuzzy soft expert set as follows.

Definition 11: A complex intuitionistic fuzzy soft expert set $(F, A)$ over $U$ is said to be empty whenever $A=\phi$.

Following the notation of Zhu and Wen (2013), we donate empty complex intuitionistic fuzzy soft expert set by $(\phi, \phi)$.
Now, we turn to define the universal set of a complex intuitionistic fuzzy soft expert $\operatorname{set}(F, A)$.

Definition 12: A complex intuitionistic fuzzy soft expert set $(F, A)$ over $U$ is said to be universal if $A=Z$ and $F\left(\left(e_{j}, w_{k}, O\right)\right):=U^{*}=\left\{\left(u_{i}, 1,0\right): u_{i} \in U\right\}$ for all $\left(e_{j}, w_{k}, O\right) \in A$.

Again, following the notation of Zhu and Wen (2013), we donate universal complex intuitionistic fuzzy soft expert set by $\left(U^{*}, Z\right)$. Note that $F\left(\left(e_{j}, w_{k}, O\right)\right)=\left\{\left(u_{i}, 1,0\right): u_{i} \in U\right\}$ means that each $u_{i} \in U$ assigns with membership grade equal 1 and nonmembership grade equal 0 . Furthermore, let us denote a set of all elements in the universal set $U$ where each element of $U$ assigns with 1-grade membership and 0 -grade nonmembership by $U^{*}$.

In the next definition, we define the subset of a complex intuitionistic fuzzy soft expert set.

Definition 13: A complex intuitionistic fuzzy soft expert set $(F, A)$ over $U$ is a subset of a complex intuitionistic fuzzy soft expert set $(G, B)$ over $U$, if $A \subseteq B$ and $F\left(\left(e_{j}, w_{k}, O\right)\right) \subseteq G\left(\left(e_{j}, w_{k}, O\right)\right)$ for all $\left(e_{j}, w_{k}, O\right) \in A$.

Subsequently, we define the equality of complex intuitionistic fuzzy soft expert set.

Definition 14: A two complex intuitionistic fuzzy soft expert sets $(F, A)$ over $U$ and complex intuitionistic fuzzy soft expert set $(G, B)$ over $U$ are said to be equal, if $A=B$ and $F\left(\left(e_{j}, w_{k}, O\right)\right)=G\left(\left(e_{j}, w_{k}, O\right)\right)$ for all $\left(e_{j}, w_{k}, O\right) \in A$.

Obviously, the empty complex intuitionistic fuzzy soft expert sets $(\phi, \phi)$ is a subset of any other complex intuitionistic fuzzy soft expert set. Also, any $(F, A)$ is a subset of the universal complex intuitionist fuzzy soft expert set $\left(U^{*}, Z\right)$. The following proposition states this remark formally.

Proposition 1: Any complex intuitionistic fuzzy soft expert set $(F, A)$ over $U$ satisfies the following inclusion:

$$
(\phi, \phi) \subseteq(F, A) \subseteq\left(U^{*}, Z\right),
$$

In the forthcoming definition, we define the intersection of two complex intuitionistic fuzzy soft expert set $(F, A)$ and $(G, B)$ over $U$.

Definition 15: An intersection of two complex intuitionistic fuzzy soft expert set $(F, A)$ and $(G, B)$ over $U$ is defined as $(F \cap G, C)$, where: 


$$
\begin{aligned}
& C=\left\{\left(e_{j}, w_{k}, O\right) \in A \cap B:\right. \\
& \left.F=\left(\left(e_{j}, w_{k}, O\right)\right) G\left(\left(e_{j}, w_{k}, O\right)\right) \neq \phi\right\}, \\
& (F \cap G)\left(\left(e_{j}, w_{k}, O\right)\right)=F\left(\left(e_{j}, w_{k}, O\right)\right) \cap G\left(\left(e_{j}, w_{k}, O\right)\right),
\end{aligned}
$$

We note here that $\mathcal{F}\left(\left(e_{j}, w_{k}, O\right)\right)$ and $G\left(\left(e_{j}, w_{k}, O\right)\right)$ are two complex intuitionistic fuzzy sets and their intersection $F\left(\left(e_{j}, w_{k}, O\right)\right)$ follows the definition and the axioms of the intersection of complex intuitionistic fuzzy soft expert sets by (Ramot et al., 2002; Alkouri and Salleh, 2012).

In the following definition we define the union of two complex intuitionistic fuzzy soft expert sets $(F, A)$ and $(G, B)$ over $U$.

Definition 16: A union of two complex intuitionistic fuzzy soft expert sets $(F, A)$ and $(G, B)$ over $U$ is defined as $(F \cup G, C)$, where:

$$
\begin{aligned}
& C=A \cup B, \\
& (F \cup G)\left(\left(e_{j}, w_{k}, O\right)\right) \\
& =\left\{\begin{array}{cc}
F\left(\left(e_{j}, w_{k}, O\right)\right) & ; \text { if }\left(e_{j}, w_{k}, O\right) \in A-B \\
G\left(\left(e_{j}, w_{k}, O\right)\right) & ; \text { if }\left(e_{j}, w_{k}, O\right) \in B-A \\
F\left(\left(e_{j}, w_{k}, O\right)\right) \cup G\left(\left(e_{j}, w_{k}, O\right)\right) & ; \text { otherwise. }
\end{array}\right.
\end{aligned}
$$

We note here that $F\left(\left(e_{j}, w_{k}, O\right)\right)$ and $G\left(\left(e_{j}, w_{k}, O\right)\right)$ are two complex intuitionistic fuzzy sets and their union $F\left(\left(e_{j}, w_{k}, O\right)\right) \cup G\left(\left(e_{j}, w_{k}, O\right)\right)$ follows the definition and the axioms of the union of complex intuitionistic fuzzy sets by (Ramot et al., 2002; Alkouri and Salleh, 2012).

We now turn to define the complement of complex intuitionistic fuzzy soft expert sets. Our approach follows Zhu and Wen (2013) where no use of so called NOT set. NOT set has its own drawbacks where its complement might be not the whole universal set. However, NOT sets have been used in literature as a pre-tool to define the complement set (Maji et al., 2001; 2003; Alkhazaleh and Salleh, 2011).

Definition 17: A complement of a complex intuitionistic fuzzy soft expert sets $(F, A)$ over $U$ with respect with universal $\left(U^{*}, Z\right)$ is defined as $(F, A)^{c}=\left(F^{c}, C\right)$, where:

$$
\begin{aligned}
& C=Z-\left\{\left(e_{j}, w_{k}, O\right) \in A: F\left(\left(e_{j}, w_{k}, O\right)\right)=U^{*}\right\} \\
& F^{c}\left(\left(e_{j}, w_{k}, O\right)\right)=\left\{\begin{array}{cc}
U^{*}-F\left(\left(e_{j}, w_{k}, O\right)\right) & ; \text { if }\left(e_{j}, w_{k}, O\right) \in A \\
U^{*} & ; \text { if otherwise }
\end{array}\right.
\end{aligned}
$$

Closely related concept of complement is the difference of two sets. The definition of the difference of two complex intuitionistic fuzzy soft expert sets is given as follows.

Definition 18: A difference of two complex intuitionistic fuzzy soft expert sets $(F, A)$ and $(G, B)$ over $U$ is defined as $(F-G, C)$, where:

$$
\begin{aligned}
& C=A-\left\{\left(e_{j}, w_{k}, O\right) \in A \cap B:\right. \\
& \left.F\left(\left(e_{j}, w_{k}, O\right)\right) \subseteq G\left(\left(e_{j}, w_{k}, O\right)\right)\right\}, \\
& (F-G)\left(\left(e_{j}, w_{k}, O\right)\right) \\
& =\left\{\begin{array}{cc}
F\left(\left(e_{j}, w_{k}, O\right)\right) \cap G\left(\left(e_{j}, w_{k}, O\right)\right) & ; \text { if }\left(e_{j}, w_{k}, O\right) \in A \cap B \\
F\left(\left(e_{j}, w_{k}, O\right)\right) & ; \text { otherwise. }
\end{array}\right.
\end{aligned}
$$

\section{Complex Intuitionistic Fuzzy Soft Expert Sets for Decision Support System Application}

This section presents a new algorithm of DSS based on CIFSES. This algorithm provides consecutive stages to find the optimal choice as shown in Fig. 1.

\section{Case Study}

Suppose a traveler wants to set a new destination among five alternative countries: France, New Zealand, South Africa, Malaysia and Jordan. The traveler bear in mind three factors of travailing: Living cost, weather and attending festivals. The traveler seeks advice from three travel consultants. In a mathematical sense, a universal set $U=\left\{u_{1}, u_{2}, u_{3}, u_{4}, u_{5}\right\}$ represents the set of alternative countries: France, New Zealand, South Africa, Malaysia and Jordan respectively. Also, a parameters set $E=\left\{e_{1}, e_{2}, e_{3}\right\}$ represents the factors: living cost, nature and attending festival respectively. Finally the travel consultants are represented by $W=\left\{w_{1}, w_{2}, w_{3}\right\}$.

Such factors require a frame where time is considered. Low season and high season affect the living cost and of course, weather changes by time and place. Likewise, attending festivals is fixed by specific time. This justifies why we need such concept (i.e., CIFSES) where complex-valued membership treats the time issue, intuitionistic fuzzy set treats the degree of belongingness and non-belongingness whereas soft expert set is parameterizing the three factors with the five alternative countries, in a complex intuitionistic fuzzy sense. Moreover, CIFSES has a place to seek advice from travel consultants. 

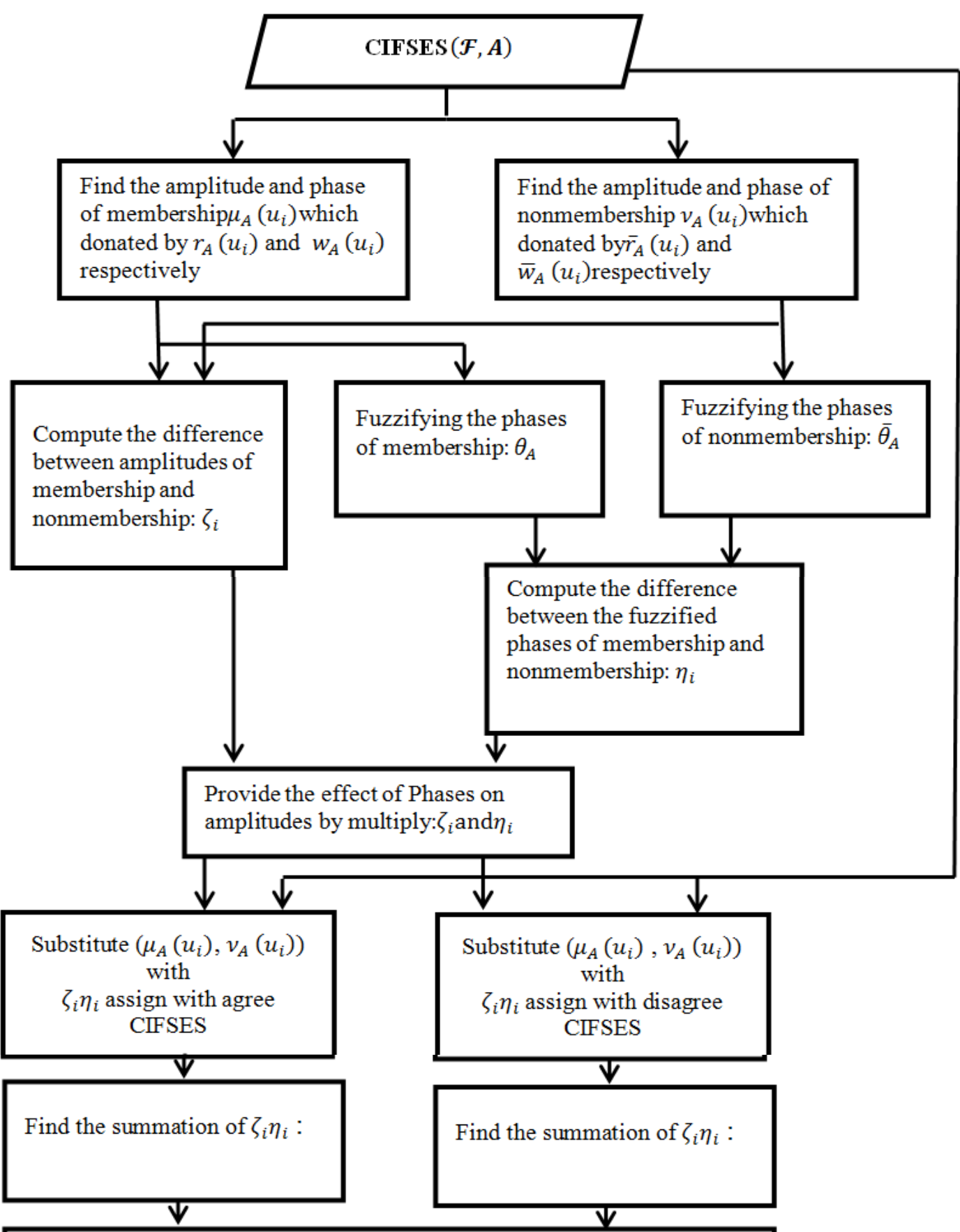

Compute the difference between agree and disagree CIFSES: $r_{i}$

ind the $\max$ of $r_{i}$

Find the max of $r_{i}$

Optimal Choice

Fig. 1: Flow chart of the proposed CIFSES- DSS algorithm 
After rating each factor based on the preference of the traveler and consults all his/her travel consultants, the traveler constructs the following CIFSE set:

$$
\begin{aligned}
& (\mathcal{F}, A)=\left\{\begin{array}{c}
\left(e_{1}, w_{1}, 1\right),\left\{\frac{u_{1}}{\left(0.2 e^{i .2 \pi}, 0.3 e^{i 0.4 \pi}\right)},\right. \\
\frac{u_{2}}{\left(0.4 e^{i 0.7 \pi}, 0.3 e^{i 0.4 \pi}\right)}, \\
\frac{u_{3}}{\left(0.3 e^{i 0.4 \pi}, 0.7 e^{i 1.1 \pi}\right)}, \\
\frac{u_{4}}{\left(0.7 e^{i .14 \pi}, 0.4 e^{i 0.2 \pi}\right)}, \\
\left.\frac{u_{5}}{\left(0.5 e^{i 1.1 \pi}, 0.4 e^{i 0.7 \pi}\right)}\right\}
\end{array}\right) \\
& \left(\begin{array}{c}
\left(e_{2}, w_{1}, 0\right),\left\{\frac{u_{1}}{\left(0.4 e^{i 1.0 \pi}, 0.5 e^{i 1.6 \pi}\right)},\right. \\
\frac{u_{2}}{\left(0.3 e^{i 0.9 \pi}, 0.4 e^{i 0.4 \pi}\right)}, \\
\frac{u_{3}}{\left(0.4 e^{i 1.4 \pi}, 0.1 e^{i 0.3 \pi}\right)}, \\
\frac{u_{4}}{\left(0.3 e^{i 1.2 \pi}, 0.6 e^{i 1.4 \pi}\right)}, \\
\left.\frac{u_{5}}{\left(0.1 e^{i 1.3 \pi}, 0.3 e^{i 0.9 \pi}\right)}\right\}
\end{array}\right), \\
& \left(e_{3}, w_{1}, 0\right),\left\{\frac{u_{1}}{\left(0.5 e^{i 0.3 \pi}, 0.5 e^{i 0.3 \pi}\right)},\right. \\
& \frac{u_{2}}{\left(0.4 e^{i 0.8 \pi}, 0.4 e^{i 0.8 \pi}\right)}, \\
& \frac{u_{3}}{\left(0.5 e^{i 1.1 \pi}, 0.5 e^{i 1.1 \pi}\right)}, \\
& \frac{u_{4}}{\left(0.4 e^{i 0.2 \pi}, 0.4 e^{i 0.2 \pi}\right)}, \\
& \left.\frac{u_{5}}{\left(0.3 e^{i 0.2 \pi}, 0.3 e^{i 0.2 \pi}\right)}\right\} \\
& \left(e_{1}, w_{2}, 1\right),\left\{\frac{u_{1}}{\left(0.7 e^{i 1.8 \pi}, 0.2 e^{i 1.3 \pi}\right)},\right. \\
& \frac{u_{2}}{\left(0.5 e^{i 1.6 \pi}, 0.2 e^{i 0.3 \pi}\right)} \text {, } \\
& \frac{u_{3}}{\left(0.2 e^{i 1.3 \pi}, 0.6 e^{i 1.5 \pi}\right)}, \\
& \frac{u_{4}}{\left(0.7 e^{i 1.6 \pi}, 0.7 e^{i 1.2 \pi}\right)}, \\
& \left.\frac{u_{5}}{\left(0.6 e^{i 1.5 \pi}, 0.5 e^{i 1.6 \pi}\right)}\right\}
\end{aligned}
$$

$$
\begin{aligned}
& \left(\begin{array}{c}
\left(e_{3}, w_{2}, 1\right),\left\{\frac{u_{1}}{\left(0.3 e^{i 0.9 \pi}, 0.9 e^{i 1.5 \pi}\right)},\right. \\
\frac{u_{2}}{\left(0.6 e^{i 1.4 \pi}, 0.9 e^{i 1.2 \pi}\right)}, \\
\frac{u_{3}}{\left(0.9 e^{i 1.2 \pi}, 0.4 e^{i 0.5 \pi}\right)}, \\
\frac{u_{4}}{\left(0.5 e^{i 0.1 \pi}, 0.3 e^{i 0.8 \pi}\right)}, \\
\left.\frac{u_{5}}{\left(0.4 e^{i 0.5 \pi}, 0.6 e^{i 1.4 \pi}\right)}\right\}
\end{array}\right\}, \\
& \left(e_{2}, w_{2}, 1\right),\left\{\frac{u_{1}}{\left(0.9 e^{i 0.7 \pi}, 0.8 e^{i 0.9 \pi}\right)},\right. \\
& \frac{u_{2}}{\left(0.7 e^{i 1.5 \pi}, 0.8 e^{i 0.9 \pi}\right)}, \\
& \frac{u_{3}}{\left(0.8 e^{i 0.9 \pi}, 0.5 e^{i 1.3 \pi}\right)}, \\
& \frac{u_{4}}{\left(0.3 e^{i .4 \pi}, 0.9 e^{i 1.6 \pi}\right)}, \\
& \left.\frac{u_{5}}{\left(0.8 e^{i 1.3 \pi}, 0.7 e^{i 0.5 \pi}\right)}\right\} \\
& \left(e_{1}, w_{3}, 0\right),\left\{\frac{u_{1}}{\left(0.6 e^{i 1.2 \pi}, 0.6 e^{i 1.3 \pi}\right)},\right. \\
& \frac{u_{2}}{\left(0.3 e^{i 1.1 \pi}, 0.6 e^{i 1.3 \pi}\right)}, \\
& \frac{u_{3}}{\left(0.6 e^{i 1.3 \pi}, 0.9 e^{i 1.3 \pi}\right)}, \\
& \frac{u_{4}}{\left(0.6 e^{i 1.0 \pi}, 0.6 e^{i 0.6 \pi}\right)}, \\
& \left.\frac{u_{5}}{\left(0.9 e^{i 1.3 \pi}, 0.3 e^{i 0.1 \pi}\right)}\right\} \\
& \left(e_{2}, w_{3}, 0\right),\left\{\frac{u_{1}}{\left(0.5 e^{i .17 \pi}, 0.7 e^{i .2 \pi}\right)},\right. \\
& \frac{u_{2}}{\left(0.2 e^{i 1.3 \pi}, 0.7 e^{i 1.2 \pi}\right)}, \\
& \frac{u_{3}}{\left(0.7 e^{i 1.2 \pi}, 0.1 e^{i 0.6 \pi}\right)}, \\
& \frac{u_{4}}{\left(0.1 e^{i 1.9 \pi}, 0.5 e^{i 1.3 \pi}\right)}, \\
& \left.\frac{u_{5}}{\left(0.1 e^{i 0.6 \pi}, 0.2 e^{i 0.6 \pi}\right)}\right\}
\end{aligned}
$$




$$
\left(\begin{array}{c}
\left(e_{3}, w_{3}, 1\right),\left\{\frac{u_{1}}{\left(0.1 e^{i 0.4 \pi}, 0.3 e^{i 0.8 \pi}\right)},\right. \\
\frac{u_{2}}{\left(0.3 e^{i 1.4 \pi}, 0.5 e^{i 0.8 \pi}\right)}, \\
\frac{u_{3}}{\left(0.5 e^{i 0.8 \pi}, 0.6 e^{i 1.4 \pi}\right)}, \\
\frac{u_{4}}{\left(0.7 e^{i 1.5 \pi}, 0.1 e^{i 1.1 \pi}\right)}, \\
\left(0.6 e^{i 1.4 \pi}, 0.3 e^{i 1.4 \pi}\right)
\end{array}\right\}
$$

This algorithm is emphasized in mathematical steps as shown below.

\section{Input: CIFSES $(F, A)$}

\section{Output: Optimal Choice (m)}

Step 1: Find $r_{A}\left(u_{i}\right)$ and $w_{A}\left(u_{i}\right)$ for membership function $\mu_{A}\left(u_{i}\right)$ for each $u_{i} \in U$,

Step 2: Find $\bar{r}_{A}\left(u_{i}\right)$ and $\bar{w}_{A}\left(u_{i}\right)$ for nonmembership function $v_{A}\left(u_{i}\right)$ for each $u_{i} \in U$,

Step 3: Compute $\zeta_{i}:=r_{A}\left(u_{i}\right)-\bar{r}_{A}\left(u_{i}\right)$ for each $u_{i} \in U$,

Step 4: Compute $\theta_{A}:=\frac{w_{A}\left(u_{i}\right)}{2 \pi}$ for each $u_{i} \in U$,
Step 5: Compute $\bar{\theta}_{A}:=\frac{\bar{w}_{A}\left(u_{i}\right)}{2 \pi}$ for each $u_{i} \in U$,

Step 6: Compute $\eta_{i}:=\theta_{A}-\bar{\theta}_{A}$ for each $u_{i} \in U$,

Step 7: Compute $\zeta_{i} \eta_{i}$ for each $i=1, \ldots, p$,

Step 8: Construct a set-valued function

$$
(\tilde{F}, A)=\left\{\left(\left(e_{j}, w_{k}, 1\right),\left\{\left(u_{i}, \zeta_{i} \eta_{i}\right): u_{i} \in U\right\}\right)\right\},
$$

Step 9: Construct set-valued function

$$
(\tilde{F}, A)=\left\{\left(\left(e_{j}, w_{k}, 0\right),\left\{\left(u_{i}, \zeta_{i} \eta_{i}\right): u_{i} \in U\right\}\right)\right\},
$$

Step 10: Find $A_{i}:=\sum \zeta_{i} \eta_{i}$ for all $i=1, \ldots, p$ for each

$$
\left(e_{j}, w_{k}, 1\right) \text {, }
$$

Step 11: Find $D_{i}:=\sum \zeta_{i} \eta_{i}$ for all $i=1, \ldots, p$ for each $\left(e_{j}, w_{k}, 0\right)$,

Step 12: Compute $r_{i}=A_{i}-D_{i}$ for each $i$

Step 13: Find $r_{m}=\max r_{i}$

Next the CIFSES $(F, A)$ is used together with a CIFSES-DSS algorithm to solve the decision-making problem stated in the case study at the beginning of this section. The following steps illustrate each step of the algorithm with correspondence table, where $(F, A)$ considered as an input to the algorithm.

Step 1: Find the amplitude $r_{A}\left(u_{i}\right)$ and phase $w_{A}\left(u_{i}\right)$ for membership function $\mu_{A}\left(u_{i}\right)$ as shown in Table 1 .

Table 1: The amplitude and phase for membership function

\begin{tabular}{llllll}
\hline & $u_{1}$ & $u_{2}$ & $u_{3}$ & $u_{4}$ & $u_{5}$ \\
\hline$\left(e_{1}, w_{1}, 1\right)$ & $0.2,1.2 \pi$ & $0.4,0.7 \pi$ & $0.3,0.4 \pi$ & $0.7,1.4 \pi$ & $0.5,1.1 \pi$ \\
$\left(e_{2}, w_{1}, 0\right)$ & $0.4,1.0 \pi$ & $0.3,0.9 \pi$ & $0.4,1.4 \pi$ & $0.3,1.2 \pi$ & $0.1,1.3 \pi$ \\
$\left(e_{3}, w_{1}, 0\right)$ & $0.5,0.3 \pi$ & $0.4,0.8 \pi$ & $0.5,1.1 \pi$ & $0.4,0.2 \pi$ & $0.3,0.2 \pi$ \\
$\left(e_{1}, w_{2}, 1\right)$ & $0.7,1.8 \pi$ & $0.5,1.6 \pi$ & $0.2,1.3 \pi$ & $0.7,1.6 \pi$ & $0.6,1.5 \pi$ \\
$\left(e_{2}, w_{2}, 1\right)$ & $0.9,0.7 \pi$ & $0.7,1.5 \pi$ & $0.8,0.9 \pi$ & $0.3,0.4 \pi$ & $0.8,1.3 \pi$ \\
$\left(e_{3}, w_{2}, 1\right)$ & $0.3,0.9 \pi$ & $0.6,1.4 \pi$ & $0.9,1.2 \pi$ & $0.5,0.1 \pi$ & $0.4,0.5 \pi$ \\
$\left(e_{1}, w_{3}, 0\right)$ & $0.6,1.2 \pi$ & $0.3,1.1 \pi$ & $0.6,1.3 \pi$ & $0.6,1.0 \pi$ & $0.9,1.3 \pi$ \\
$\left(e_{2}, w_{3}, 0\right)$ & $0.5,1.7 \pi$ & $0.2,1.3 \pi$ & $0.7,1.2 \pi$ & $0.1,1.9 \pi$ & $0.1,0.6 \pi$ \\
$\left(e_{3}, w_{3}, 1\right)$ & $0.1,0.4 \pi$ & $0.3,1.4 \pi$ & $0.5,0.8 \pi$ & $0.7,1.5 \pi$ & $0.6,1.4 \pi$ \\
\hline
\end{tabular}

Step 2: Find the amplitude $\bar{r}_{A}\left(u_{i}\right)$ and phase $\bar{w}_{A}\left(u_{i}\right)$ for nonmembership function $v_{A}\left(u_{i}\right)$ as shown in Table 2.

Table 2: The amplitude and phase for nonmembership function

\begin{tabular}{llllll}
\hline & $u_{1}$ & $u_{2}$ & $u_{3}$ & $u_{4}$ & $u_{5}$ \\
\hline$\left(e_{1}, w_{1}, 1\right)$ & $0.3,0.4 \pi$ & $0.3,0.4 \pi$ & $0.7,1.1 \pi$ & $0.4,0.2 \pi$ & $0.4,0.7 \pi$ \\
$\left(e_{2}, w_{1}, 0\right)$ & $0.5,1.6 \pi$ & $0.4,0.4 \pi$ & $0.1,0.3 \pi$ & $0.6,1.4 \pi$ & $0.3,0.9 \pi$ \\
$\left(e_{3}, w_{1}, 0\right)$ & $0.6,1.1 \pi$ & $0.5,1.1 \pi$ & $0.3,1.2 \pi$ & $0.5,1.0 \pi$ & $0.4,0.8 \pi$ \\
$\left(e_{1}, w_{2}, 1\right)$ & $0.2,1.3 \pi$ & $0.2,0.3 \pi$ & $0.6,1.5 \pi$ & $0.7,1.2 \pi$ & $0.5,1.6 \pi$ \\
$\left(e_{2}, w_{2}, 1\right)$ & $0.8,0.9 \pi$ & $0.8,0.9 \pi$ & $0.5,1.3 \pi$ & $0.9,1.6 \pi$ & $0.7,0.5 \pi$ \\
$\left(e_{3}, w_{2}, 1\right)$ & $0.9,1.5 \pi$ & $0.9,1.2 \pi$ & $0.4,0.5 \pi$ & $0.3,0.8 \pi$ & $0.6,1.4 \pi$ \\
$\left(e_{1}, w_{3}, 0\right)$ & $0.6,1.3 \pi$ & $0.6,1.3 \pi$ & $0.9,1.3 \pi$ & $0.6,0.6 \pi$ & $0.3,0.1 \pi$ \\
$\left(e_{2}, w_{3}, 0\right)$ & $0.7,1.2 \pi$ & $0.7,1.2 \pi$ & $0.1,0.6 \pi$ & $0.5,1.3 \pi$ & $0.2,0.6 \pi$ \\
$\left(e_{3}, w_{3}, 1\right)$ & $0.3,0.8 \pi$ & $0.5,0.8 \pi$ & $0.6,1.4 \pi$ & $0.1,1.1 \pi$ & $0.3,1.4 \pi$ \\
\hline
\end{tabular}


Step 3: Compute $\zeta_{i}:=r_{A}\left(u_{i}\right)-\bar{r}_{A}\left(u_{i}\right)$ for each $u_{i} \in U$ as shown in Table 3.

Table 3: The value of $\zeta_{i}$

\begin{tabular}{llllll}
\hline & $u^{1}$ & $u^{2}$ & $u^{3}$ & $u^{4}$ & $u^{5}$ \\
\hline$\left(e_{1}, w_{1}, 1\right)$ & -0.1 & 0.1 & -0.4 & 0.3 & 0.1 \\
$\left(e_{2}, w_{1}, 0\right)$ & -0.1 & -0.1 & 0.3 & -0.3 & -0.2 \\
$\left(e_{3}, w_{1}, 0\right)$ & -0.1 & -0.1 & -0.2 & -0.1 & -0.1 \\
$\left(e_{1}, w_{2}, 1\right)$ & 0.5 & 0.3 & -0.4 & 0.0 & 0.1 \\
$\left(e_{2}, w_{2}, 1\right)$ & 0.1 & 0.1 & 0.3 & -0.6 & 0.1 \\
$\left(e_{3}, w_{2}, 1\right)$ & -0.6 & -0.3 & 0.5 & 0.2 & -0.2 \\
$\left(e_{1}, w_{3}, 0\right)$ & 0.0 & -0.3 & -0.3 & 0.0 & 0.6 \\
$\left(e_{2}, w_{3}, 0\right)$ & -0.2 & -0.5 & 0.6 & -0.4 & -0.1 \\
$\left(e_{3}, w_{3}, 1\right)$ & -0.2 & -0.2 & -0.1 & 0.6 & 0.3 \\
\hline
\end{tabular}

Step 4: Compute $\theta_{A}:=w_{A}\left(u_{i}\right) / 2 \pi$ for each $u_{i} \in U$ as shown in Table 4 .

Table 4: The value of $\theta_{A}$

\begin{tabular}{llllll}
\hline & $u_{1}$ & $u_{2}$ & $u_{3}$ & $u_{4}$ & $u_{5}$ \\
\hline$\left(e_{1}, w_{1}, 1\right)$ & 0.6 & 0.35 & 0.2 & 0.7 & 0.55 \\
$\left(e_{2}, w_{1}, 0\right)$ & 0.5 & 0.45 & 0.7 & 0.6 & 0.65 \\
$\left(e_{3}, w_{1}, 0\right)$ & 0.15 & 0.4 & 0.55 & 0.1 & 0.1 \\
$\left(e_{1}, w_{2}, 1\right)$ & 0.9 & 0.8 & 0.65 & 0.2 & 0.75 \\
$\left(e_{2}, w_{2}, 1\right)$ & 0.35 & 0.75 & 0.45 & 0.05 & 0.65 \\
$\left(e_{3}, w_{2}, 1\right)$ & 0.45 & 0.7 & 0.6 & 0.5 & 0.25 \\
$\left(e_{1}, w_{3}, 0\right)$ & 0.6 & 0.55 & 0.65 & 0.95 & 0.65 \\
$\left(e_{2}, w_{3}, 0\right)$ & 0.85 & 0.65 & 0.6 & 0.75 & 0.3 \\
$\left(e_{3}, w_{3}, 1\right)$ & 0.2 & 0.7 & 0.4 & 0.7 \\
\hline
\end{tabular}

Step 5: Compute $\bar{\theta}_{A}:=\bar{w}_{A}\left(u_{i}\right) / 2 \pi$ for each $u_{i} \in U$ as shown in Table 5.

Table 5: The value of $\bar{\theta}_{A}$

\begin{tabular}{llllll}
\hline & $u_{1}$ & $u_{2}$ & $u_{3}$ & $u_{4}$ & $u_{5}$ \\
\hline$\left(e_{1}, w_{1}, 1\right)$ & 0.4 & 0.2 & 0.55 & 0.1 & 0.35 \\
$\left(e_{2}, w_{1}, 0\right)$ & -0.3 & 0.2 & 0.15 & 0.7 & 0.45 \\
$\left(e_{3}, w_{1}, 0\right)$ & -0.4 & 0.55 & 0.6 & 0.5 & 0.6 \\
$\left(e_{1}, w_{2}, 1\right)$ & 0.25 & 0.15 & 0.75 & 0.8 & 0.8 \\
$\left(e_{2}, w_{2}, 1\right)$ & -0.1 & 0.45 & 0.65 & 0.4 & 0.25 \\
$\left(e_{3}, w_{2}, 1\right)$ & -0.3 & 0.6 & 0.25 & 0.3 & 0.7 \\
$\left(e_{1}, w_{3}, 0\right)$ & -0.05 & 0.65 & 0.65 & 0.65 & 0.05 \\
$\left(e_{2}, w_{3}, 0\right)$ & 0.25 & 0.6 & 0.3 & 0.55 & 0.3 \\
$\left(e_{3}, w_{3}, 1\right)$ & -0.2 & 0.4 & 0.7 & 0.7 \\
\hline
\end{tabular}

Step 6: Compute $\eta_{i}:=\theta_{A}-\bar{\theta}_{A}$ for each $u_{i} \in U$ as shown in Table 6.

Table 6: The value of $\eta_{i}$

\begin{tabular}{llllll}
\hline & $u_{1}$ & $u_{2}$ & $u_{3}$ & $u_{4}$ & $u_{5}$ \\
\hline$\left(e_{1}, w_{1}, 1\right)$ & 0.2 & -0.05 & -0.35 & 0.6 & 0.2 \\
$\left(e_{2}, w_{1}, 0\right)$ & 0.8 & 0.75 & 0.55 & -0.1 & -0.4 \\
$\left(e_{3}, w_{1}, 0\right)$ & 0.55 & 0.8 & -0.05 & 0.2 & -0.3 \\
$\left(e_{1}, w_{2}, 1\right)$ & 0.65 & 0.55 & -0.1 & -0.6 & -0.05 \\
$\left(e_{2}, w_{2}, 1\right)$ & 0.45 & 0.85 & -0.2 & -0.35 & 0.40 \\
$\left(e_{3}, w_{2}, 1\right)$ & 0.75 & 1.00 & 0.35 & 0.2 & -0.45 \\
$\left(e_{1}, w_{3}, 0\right)$ & 0.65 & 0.6 & 0.00 & 0.3 & 0.6 \\
$\left(e_{2}, w_{3}, 0\right)$ & 0.6 & 0.4 & 0.30 & 0.2 & 0.0 \\
$\left(e_{3}, w_{3}, 1\right)$ & 0.4 & 0.9 & -0.3 & 0.0 \\
\hline
\end{tabular}

Step 7: Provide the effect of phases on amplitudes by multiply $\zeta_{i}$ and $\eta_{i}$ as shown in Table 7 . 
Table 7: The value of $\zeta_{i} \eta_{i}$

\begin{tabular}{lllll}
\hline & $u_{1}$ & $u_{2}$ & $u_{3}$ & $u_{5}$ \\
\hline$\left(e_{1}, w_{1}, 1\right)$ & -0.02 & -0.005 & 0.14 & 0.02 \\
$\left(e_{2}, w_{1}, 0\right)$ & -0.08 & -0.075 & 0.165 & -0.04 \\
$\left(e_{3}, w_{1}, 0\right)$ & -0.055 & -0.08 & 0.01 & 0.03 \\
$\left(e_{1}, w_{2}, 1\right)$ & 0.325 & 0.165 & 0.04 & 0.04 \\
$\left(e_{2}, w_{2}, 1\right)$ & 0.045 & 0.085 & -0.06 & 0.00 \\
$\left(e_{3}, w_{2}, 1\right)$ & -0.45 & -0.3 & 0.175 & 0.36 \\
$\left(e_{1}, w_{3}, 0\right)$ & 0.00 & -0.18 & 0.00 & -0.07 \\
$\left(e_{2}, w_{3}, 0\right)$ & -0.12 & -0.2 & 0.00 & 0.00 \\
$\left(e_{3}, w_{3}, 1\right)$ & -0.08 & -0.18 & 0.03 & -0.12 \\
\hline
\end{tabular}

Step 8: Construct agree CIFSESs for each $u_{i}$ as shown in Table 8.

Table 8: Agree of CIFSESs

\begin{tabular}{llllll} 
& $u_{1}$ & $u_{2}$ & $u_{3}$ & $u_{4}$ & \multicolumn{1}{c}{$u_{5}$} \\
\hline$\left(e_{1}, w_{1}, 1\right)$ & -0.02 & -0.005 & 0.14 & 0.18 & 0.02 \\
$\left(e_{1}, w_{2}, 1\right)$ & 0.325 & 0.165 & 0.04 & 0.00 & -0.005 \\
$\left(e_{2}, w_{2}, 1\right)$ & 0.045 & 0.085 & -0.06 & 0.36 & 0.04 \\
$\left(e_{3}, w_{2}, 1\right)$ & -0.45 & -0.3 & 0.175 & -0.07 & 0.09 \\
$\left(e_{3}, w_{3}, 1\right)$ & -0.08 & -0.18 & 0.03 & 0.12 & 0.00 \\
$u_{i}$ Score & -0.18 & -0.235 & 0.325 & 0.59 & 0.145 \\
\hline
\end{tabular}

Step 9: Construct disagree CIFSESs for each $u_{i}$ as shown in Table 9.

Table 9: Disagree of CIFSESs

\begin{tabular}{llllll}
\hline & $u_{1}$ & $u_{2}$ & $u_{3}$ & $u_{4}$ & $u_{5}$ \\
\hline$\left(e_{2}, w_{1}, 0\right)$ & -0.08 & -0.075 & 0.165 & 0.03 & -0.04 \\
$\left(e_{3}, w_{1}, 0\right)$ & -0.055 & -0.08 & 0.01 & 0.04 & 0.03 \\
$\left(e_{1}, w_{3}, 0\right)$ & 0.00 & -0.18 & 0.00 & 0.00 & 0.36 \\
$\left(e_{2}, w_{3}, 0\right)$ & -0.12 & -0.2 & 0.18 & -0.12 & 0.00 \\
$u_{i}$ Score & -0.115 & -0.535 & 0.355 & -0.05 & 0.35 \\
\hline
\end{tabular}

Step 10: Find $A_{i}:=\sum \zeta_{i} \eta_{i}$ for all $i=1, \ldots, p$ for each $\left(e_{j}, w_{k}, 1\right)$ as shown in Table 10

Table 10: Score of agree CIFSESs

\begin{tabular}{llllll}
\hline & $u_{1}$ & $u_{2}$ & $u_{3}$ & $u_{4}$ & $u_{5}$ \\
\hline$\left(e_{1}, w_{1}, 1\right)$ & -0.02 & -0.005 & 0.14 & 0.18 & 0.02 \\
$\left(e_{1}, w_{2}, 1\right)$ & 0.325 & 0.165 & 0.04 & 0.00 & -0.005 \\
$\left(e_{2}, w_{2}, 1\right)$ & 0.045 & 0.085 & -0.06 & 0.36 & 0.04 \\
$\left(e_{3}, w_{2}, 1\right)$ & -0.45 & -0.3 & 0.175 & -0.07 & 0.09 \\
$\left(e_{3}, w_{3}, 1\right)$ & -0.08 & -0.18 & 0.03 & 0.12 & 0.00 \\
$u_{i}$ Score & -0.18 & -0.235 & 0.325 & 0.59 & 0.145 \\
\hline
\end{tabular}

Step 11: Find $D_{i}:=\sum \zeta_{i} \eta_{i}$ for all $i=1, \ldots, p$ for each $\left(e_{j}, w_{k}, 0\right)$ as shown in Table 11.

Table 11: Score of disagree CIFSESs

\begin{tabular}{llllll}
\hline & $u_{1}$ & $u_{2}$ & $u_{3}$ & $u_{4}$ & $u_{5}$ \\
\hline$\left(e_{2}, w_{1}, 0\right)$ & -0.08 & -0.075 & 0.165 & 0.03 & -0.04 \\
$\left(e_{3}, w_{1}, 0\right)$ & -0.055 & -0.08 & 0.01 & 0.04 & 0.03 \\
$\left(e_{1}, w_{3}, 0\right)$ & 0.00 & -0.18 & 0.00 & 0.00 & 0.36 \\
$\left(e_{2}, w_{3}, 0\right)$ & -0.12 & -0.2 & 0.18 & -0.12 & 0.00 \\
$u_{i}$ Score & -0.115 & -0.535 & 0.355 & -0.05 & 0.35 \\
\hline
\end{tabular}


Step 12: Compute the difference between agree and disagree CIFSES: $r_{i}=A_{i}-D_{i}$ as shown in Table 12 .

Table 12: The value the of difference between agree score and disagree score

\begin{tabular}{llll}
\hline & $A_{i}$ & $D_{i}$ & $r_{i}$ \\
\hline Score $u_{1}$ & -0.180 & -0.115 & -0.065 \\
Score $u_{2}$ & -0.235 & -0.535 & 0.300 \\
Score $u_{3}$ & 0.325 & 0.355 & -0.030 \\
Score $u_{4}$ & 0.590 & -0.050 & 0.640 \\
Score $u_{5}$ & 0.145 & 0.350 & -0.205 \\
\hline
\end{tabular}

Step 13: Find the max of $r_{i}: r_{m}=\max r_{i}$ as shown in Table 13.

Table 13: The value the of difference between agree score and disagree score

\begin{tabular}{lc}
\hline & $r_{i}$ \\
\hline Score $u_{1}$ & -0.065 \\
Score $u_{2}$ & 0.300 \\
Score $u_{3}$ & -0.030 \\
Score $\boldsymbol{u}_{\mathbf{4}}$ & $\mathbf{0 . 6 4 0}$ \\
Score $u_{5}$ & -0.205 \\
$\max r_{i}$ & $\mathbf{0 . 6 4 0}$ \\
\hline
\end{tabular}

Therefore, the optimal choice is $u_{4}$ which present Malaysia. Comparing with all algorithms in the Literature Reviews, our algorithm considers the overall performance of all alternatives whereas their algorithms eliminate those alternatives without highest grads.

\section{Conclusion}

In this study, the concept of complex intuitionistic fuzzy soft expert set has been established. The basic operations on complex intuitionistic fuzzy soft expert sets, namely the intersection, union, complement and difference have been derived. Finally, a proposed CIFSES-DSS algorithm has been applied to solve hypothetical DM problems. As a future work, it would be interesting to implement the CIFSES-DSS algorithm in several sectors.

\section{Acknowledgement}

We thank the anonymous referees for their usful suggestions.

\section{Author's Contributions}

All authors equally contributed to this work.

\section{Ethics}

There are no ethical issues with this article.

\section{References}

Alhazaymeh, K. and N. Hassan, 2013. Generalized interval-valued vague soft set. Applied Math. Sci., 7: 6983-6988. DOI: 10.12988/ams.2013.310575

Ali, M., D.E. Tamir N.D. Rishe and A. Kandel, 2016. Complex intuitionistic fuzzy classes. IEEE Proceedings of the International Conference on Fuzzy Systems, IEEE Xplore Press, Vancouver, Jul. 24-29, pp: 2027-2034. DOI: 10.1109/FUZZ-IEEE.2016.7737941

Alkhazaleh, S. and A.R. Salleh, 2011. Soft expert sets. Adv. Dec. Sci., 2011, 11-12. DOI: $10.1155 / 2011 / 757868$

Alkhazaleh, S. and A.R. Salleh, 2012. Generalised interval-valued fuzzy soft set. J. Applied Math., 2012, 11-18. DOI: 10.1155/2012/870504

Alkhazaleh, S., A.R. Salleh and N. Hassan, 2011. Possibility fuzzy soft set. Adv. Decision Sci., 2011: 1-18. DOI: $10.1155 / 2011 / 479756$

Alkouri, A.S. and A.R. Salleh, 2012. Complex intuitionistic fuzzy sets. AIP Conf. Proc., 1482: 464-470. DOI: 10.1063/1.4757515

Alkouri, A.U.M. and A.R. Salleh, 2013. Complex atanassov's intuitionistic fuzzy relation. Abstract Applied Anal., 2013, 1-18.

DOI: $10.1155 / 2013 / 287382$

Al-Qudah, Y. and N. Hassan, 2017. Bipolar fuzzy soft expert set and its application in decision making. Int. J. Applied Dec. Sci., 10: 175-191.

DOI: 10.1504/IJADS.2017.084310

Al-Quran, A. and N. Hassan, 2016. Fuzzy parameterised single valued neutrosophic soft expert set theory and its application in decision making. Int. J. Applied Dec. Sci., 9: 212-227.

DOI: $10.1504 /$ IJADS.2016.080121

Atanassov, K.T., 1986. Intuitionistic fuzzy sets. Fuzzy Sets Syst., 20: 87-96. DOI: 10.1016/S0165-0114(86)80034-3

Bashir, M. and A.R. Salleh, 2012a. Fuzzy parameterized soft expert set. Abstract Applied Analysis, 2012: 115. DOI: $10.1155 / 2012 / 258361$

Bashir, M. and A.R. Salleh, 2012b. Possibility fuzzy soft expert set. Open J. Appl. Sci., 2: 208-211. DOI: 10.4236/ojapps.2012.24B047

Bashir, M., A.R. Salleh and S. Alkhazaleh, 2012. Possibility intuitionistic fuzzy soft set. Advances Decision Sci., 2012, 1-24. DOI: $10.1155 / 2012 / 404325$

Broumi, S. and F. Smarandache, 2015. Single valued neutrosophic soft expert sets and their application in decision making. J. New Theory, 3: 67-88.

Hazaymeh, A.A., I.B. Abdullah Z.T. Balkhi and R.I. Ibrahim, 2012. Generalized fuzzy soft expert set. J. Applied Math., 2012, 1-22.

DOI: $10.1155 / 2012 / 328195$ 
Jemal, H., Z. Kechaou and M. Ben Ayed, 2017. Enhanced decision support systems in intensive care unit based on intuitionistic fuzzy sets. Advances Fuzzy Systems, 2017: 1-8. DOI: $10.1155 / 2017 / 7371634$

Jun, M., Z. Guangquan and L. Jie, 2012. A method for multiple periodic factor prediction problems using complex fuzzy sets. IEEE Trans. Fuzzy Syst., 20: 32-45. DOI: 10.1109/TFUZZ.2011.2164084

Karaaslan, F., 2014. Possibility neutrosophic soft sets with applications in decision making and similarity measure. arXiv preprint arXiv:1407.3211.

Karacapilidis, N., 2006. An overview of future challenges of decision support technologies. Springer, London.

Kumar, T. and R.K. Bajaj, 2014. On complex intuitionistic fuzzy soft sets with distance measures and entropies. J. Math., 2014: 1-12. DOI: $10.1155 / 2014 / 972198$

Maji, P., R. Biswas and A. Roy, 2003. Soft set theory. Comp. Math. Appl., 45: 555-562. DOI: $10.1016 / \mathrm{S} 0898-1221(03) 00016-6$

Maji, P.K., R. Biswas and A. Roy, 2001. Fuzzy soft sets. J. Fuzzy Math., 9: 589-602.

Molodtsov, D., 1999. Soft set theory-first results. Comp. Math. Applic., 37: 19-31. DOI: $10.1016 / \mathrm{S} 0898-1221(99) 00056-5$

Power, D.J. and R. Sharda, 2009. Decision support systems. Springer handbook of automation. Springer, Berlin, Heidelberg.
Ramot, D., R. Milo M. Friedman and A. Kandel, 2002. Complex fuzzy sets. IEEE Trans. Fuzzy Syst., 10: 171-186. DOI: 10.1109/91.995119

Sahin, M., S. Alkhazaleh and V. Ulucay, 2015. Neutrosophic soft expert sets. Appl. Math., 6: 116-127. DOI: $10.4236 / \mathrm{am} .2015 .61012$

Selvachandran, G. and A.R. Salleh, 2015. Possibility intuitionistic fuzzy soft expert set theory and its application in decision making. Int. J. Math. Mathem.. Sci., 2015, 1-11.

DOI: $10.1155 / 2015 / 314285$

$\mathrm{Xu}$, J., Y. Sun and D. Li, 2010. Intuitionistic fuzzy soft set. Proceedings of the 2nd International Workshop on Intelligent Systems and Applications, May 22-23, IEEE Xplore Press, Wuhan, China. DOI: 10.1109/IWISA.2010.5473444

Zadeh, L.A., 1965. Fuzzy sets. Inform. Control, 8: 338-353. DOI: 10.1016/S0019-9958(65)90241-X

Zhu, P. and Q. Wen, 2013. Operations on soft sets revisited. J. Applied Math., 7: 1-7. DOI: $10.1155 / 2013 / 105752$

Zimmermann, H.J., 1998. Fuzzy Decision Support Systems. Computational Intelligence: Soft Computing and Fuzzy-Neuro Integration with Applications. Berlin, Heidelberg: Springer. 